\title{
The "mirror" and the "mask": Self-focused attention, evaluation anxiety, and the recognition of psychological implications
}

\author{
STEPHEN J. DOLLINGER, LEILANI GREENING, and KAREN LLOYD \\ Southern Illinois University, Carbondale, Illinois
}

\begin{abstract}
It was predicted that attention focused on the self would interfere with performance on a task that requires recognizing the psychological implications of word associations. In particular, it was expected that the combination of a high level of test anxiety or self-consciousness and experimental testing conditions (i.e., instructions about task importance, unveiling of a mirror, and request for permission to videotape) would produce especially poor performance. Results revealed a main effect of testing condition such that subjects in the experimental condition were less vigilant and less successful in their performance than were subjects in the control condition. The results are discussed in terms of Scheibe's (1979) concept of the interpersonal prediction mode of sagacity and the "mirror" as a tool for avoiding prediction.
\end{abstract}

Psychologists who supervise clinical interviews can easily imagine observing a novice whose interview is going poorly. Despite better than average intelligence and social skill, the student interviewer may not grasp the interviewee's "real agenda" and may overlook several potentially important cues. Consequently, the interviewer may struggle through unproductive lines of questioning and awkward silences. At the next supervision meeting, the student may have insightful ideas about what took place. For various reasons, however, this student had a hard time being psychologically minded in the interview. One might hypothesize that the novice interviewer had experienced evaluation anxiety due to the supervisor's observation or possibly that the novice was self-focused to an excessive degree. This anxiety may derive from a chronic (dispositional) self-consciousness or from the fact that the novice has not been desensitized to the selffocusing effect of the mirror or to being videotaped.

This clinical scenario provides a heuristic analogy for studying the effects of self-focus and evaluation anxiety on a task that requires subject-judges to read between the lines of others' word associations. A recent series of studies using this task has shown that, without special training, many high school and college students can successfully detect which target persons have imagined committing a theft (Dollinger, 1985; Dollinger, Greening, \& Tylenda, 1985; Dollinger, Reader, Marnett, \& Tylenda, 1983; Dollinger \& Riger, 1984). (Theft is used in this instance to represent an action about which the target person might feel conflict and a wish to conceal information.) Thus, the task creates a situation whereby judges must penetrate the target persons' "masks." (See Scheibe,

Address correspondence to Stephen J. Dollinger, Department of Psychology, Southern Illinois University, Carbondale, IL 62901.
1979 , for a discussion of the interpersonal function of the mask metaphor.)

Our research has shown that the most successful subjects have a better appreciation of valid stimulus-response cues in the task: They are more likely to report using only the word clues (Dollinger \& Riger, 1984); they are better able to identify valid diagnostic clues (Dollinger, 1985); and they seem to be more alert to valid clues while considering the information (Dollinger et al., 1985). In fact, the more successful subjects may be generally more vigilant at the time of testing (Dollinger \& Riger, 1984). These findings support the conclusion that the task involves what Scheibe $(1978,1979)$ has termed "the prediction mode of sagacity." Sagacious predictions are those based on good observation, careful reasoning, and "a knowledge of relationships... of what goes with what" $(1979$, p. 14) in terms of the stimuli functionally related to a criterion of interest.

Given that successful reading between the lines requires alertness to empirical cues in this task, an important corollary arises: Presumably, any factor that causes the subjectjudge to redirect attention from task-cues to the self should interfere with performance. Thus, evaluation apprehension or test anxiety is likely to disrupt reading between the lines, because of both its narrowing effect on cue utilization (Geen, 1976, 1980) and its simultaneous effect of redirecting attention to the self (Wine, 1982). In addition, to the extent that a subject is self-reflective or is concerned about his/her appearance to another (i.e., privately or publicly self-conscious), that subject may not be attending to important cues in the task. Thus, self-consciousness (Fenigstein, Scheier, \& Buss, 1975) might also play a role.

In sum, it was hypothesized that high self-consciousness and test anxiety would interfere with success at the present judgment task. Because these dispositions may require 
eliciting cues to have an influence, the judgment task was administered under two conditions. For one group, the standard instructions were used as a control condition. For the other group, experimental conditions were designed to arouse evaluation anxiety in test-anxious subjects and self-awareness in self-conscious subjects. The manipulation consisted of instructions about the importance of the task, the unveiling of a mirror, and a request for permission to videotape. A comparison of performance in the experimental and control conditions also provides a useful experimental analogue to the predicted effects for personality variables. Our main prediction, however, was that personality and test condition would interact so that highly anxious (or self-conscious) subjects in the experimental condition would do especially poorly at recognizing implications.

\section{METHOD}

\section{Subjects}

Thirty male and 30 female college students participated in exchange for payment or for extra credit in psychology courses. Subjects were recruited from undergraduate psychology and criminal justice classes and by way of announcements posted in dormitories. By chance, the spring wave of data collection yielded the planned sample of male subjects but only one third of the desired number of female subjects. Recruitment during the summer term added 20 women to the sample. Subjects ranged in age from 17 to 45 years (mean $=20.4, S D=3.9$ ).

\section{Design}

The experimental design for analyses consisted of $2 \times 2 \times 2$ cross classifications of testing condition (control, experimental), personality variable (high, low), and gender. Four analyses were conducted, each with one of the personality measures serving to define the second classification variable based on a median split. The 37-item Text Anxiety Scale (Sarason, 1972, 1980) and the 23-item Self-Consciousness Scale (Fenigstein et al., 1975) served as the personality measures in this study. The latter scale measures private and public self-consciousness as well as social anxiety. Cutoff scores for inclusion in the high groups were 16, 13,24 , and 20 on test anxiety, social anxiety, and private and public self-consciousness, respectively.

\section{Experimental Tasks}

Judgment task. The first experimental task presented a series of 20 word-association stimuli, each followed by the word-association responses of three target persons (comprising a triad) to be judged. Within each of 10 triads, one of the target persons, prior to taking the wordassociation test in a videotaped session, had imagined committing a theft (in accord with a randomly assigned imaginary script). In this study we used a booklet version of the task, transcribed from the videotape. Thus, the task required that the subject read between the suspects' associations in an attempt to identify the thief. This version of the task and the original videotaped version have been used together in several studies yielding similar results (Dollinger, 1985; Dollinger et al., 1985). One dependent measure on this task consisted of the number of correct judgments (hits) on the 10 triads. The second measure (strategy use) consisted of the number of triads on which subjects used a vigilant taskoriented strategy of circling ostensibly diagnostic word associations, as permitted by instructions. In a previous study, subjects who used this strategy were more successful on the judgment task and more aware of valid clues (see below) in the judgment task (Dollinger et al., 1985).

Clue-awareness task. In the second experimental task, subjects were presented with a list of 24 word-association stimulus-response pairs based on the first task. Eight of these pairs were significantly diagnostic of suspect status as a thief, four were contradiagnostic of thief status, and 12 were nondiagnostic (Dollinger, 1985). Subjects attempted to iden- tify which pairs were diagnostic, contradiagnostic, and nondiagnostic, with dependent measures consisting of the number of correct judgments of these three types. A fourth measure, clue errors, consisted of the number of diagnostic word pairs thought to be contradiagnostic and vice versa.

\section{Procedure}

Subject groups ranged in size from 1 to 5 persons. Testing was conducted in a small classrom containing a one-way observation mirror along one wall. Before subjects arrived, the mirror was covered with paper. Subjects took seats in a row positioned about $3 \mathrm{ft}$ away from and directly facing the concealed mirror. After subjects had been informed about the general nature of the experiment, they completed the Test Anxiety and Self-Consciousness scales.

Next, subjects received a copy of the judgment task booklet. They were asked to imagine their own associations to the stimulus words, read the thief script, and then again imagine their most likely associations. Subjects then completed the judgment task and the clue-awareness task. This sequence constituted the control condition.

Prior to beginning the judgment task, subjects in the experimental condition were informed that the task was considered by the researchers to predict intelligence, social skill, and success in certain occupations. They also were informed that the researchers suspected that "body language is a moderating variable" in the task and, as a result, "it is necessary to request permission to videotape you while taking the task so that, at a later time, a team of psychologists can evaluate your performance more fully." After subjects signed informed consent documents to permit videotaping, the mirror was unveiled. (No subjects refused, although this option was available.) At this point, subjects in the experimental group took part in the judgment task and then the clue-awareness task. The experiment concluded with a debriefing.

Subjects taking part during the summer term (all women) were asked to complete several additional questionnaires about their experiences during the experiment. These included Sarason's (1980) Cognitive Interference Questionnaire and a supplementary 14-item cognitions checklist devised for the experiment, questionnaires to assess memory for task details, and an adjective checklist of current mood state (based on Zuckerman \& Lubin, 1965)

\section{RESULTS}

\section{Main Analyses}

A series of four $2 \times 2 \times 2$ least squares analyses of variance (ANOVAs) were conducted by cross classifying condition, gender, and personality variable. Each ANOVA used a personality classification (high/low) on one of the four variables: test anxiety, private self-consciousness, public self-consciousness, or social anxiety. Dependent variables consisted of performance on judgment task, use of the vigilant strategy, and scores on the clue-awareness task. Two consistent condition main effects indicated that subjects in the control condition used the vigilant strategy more and were more successful on the judgment task than subjects in the experimental condition. For strategy use, the main effect of condition was significant in all four ANOVAs, with $F(1,52)$ ranging from 7.24 to 8.92 (all $p s<.01$ ). For judgment, the main effect $F$ ratio ranged from $3.08(p<.10)$ to $6.38(p<.02)$. The effect was statistically significant in two ANOVAs and marginally significant in the two others. Descriptive statistics for these effects are presented in Table 1.

The strategy-use measure also yielded a significant triple interaction in one ANOVA, condition $\times$ gender $\times$ private self-consciousness $[F(1,52)=6.80, p<.02]$. 
Table 1

Descriptive Statistics for the Main Effect of Testing Condition on Strategy Use and Judgment Performance

\begin{tabular}{lcccccc}
\hline & \multicolumn{4}{c}{ Condition } \\
\cline { 2 - 3 } \multicolumn{1}{c}{ Measure } & \multicolumn{2}{c}{ Control } & & \multicolumn{2}{c}{ Experimental } \\
\cline { 2 - 3 } \cline { 5 - 7 } & Mean & $S D$ & & Mean & $S D$ \\
\hline Vigilant strategy use & 5.4 & 4.8 & & 2.1 & 3.9 \\
Judgment performance & 5.9 & 1.7 & & 5.0 & 2.0 \\
\hline
\end{tabular}

Note $-N=30$ subjects per condition. $S D=$ standard deviation. Scores on both measures have a maximum possible value of 10 .

The main effect trend held true for all combinations of gender and self-consciousness. However, within the experimental group, women who had scored low on private self-consciousness were more likely to use vigilant strategy than were men or highly self-conscious women. Additionally, a gender $\times$ social anxiety interaction obtained for this measure $[F(1,52)=4.50, p<.05]$. Lowanxiety women and high-anxiety men were particularly vigilant.

The only significant effect of clue-awareness measures was the condition $\times$ social anxiety interaction on errors $[F(1,52)=4.37, p<.05]$. Highly anxious subjects in the control group made more errors in judging the diagnosticity of word-association clues than did low-anxiety subjects in the control group or all subjects in the experimental group.

\section{Supplementary Analyses}

Two items from Sarason's (1980) Cognitive Interference Questionnaire showed significant group differences in ANOVAs conducted to explore condition and condition $X$ personality effects. Subjects in the experimental group were more likely than those in the control group to be concerned with what the experimenter thought of them [means $=1.6$ vs. $2.6(p<.05$ in three of four ANOVAs)] and to think that they should be working more carefully [means $=1.7 \mathrm{vs.} 2.8(p<.05$ in three of four ANOVAs)]; one interaction showed that subjects in the experimental group who had high public selfconsciousness scores were especially likely to think about the experimenter's thoughts about them $(p<.01)$.

On the supplementary cognition checklist, subjects in both groups typically endorsed few of the thoughts irrelevant to the judgment task but most of the relevant ones. The last item of this checklist was designed as a manipulation check for attention to the mirror: "[thought about] who/what was behind the mirror." None of the control group subjects thought about this by definition, whereas $82 \%$ of the experimental group subjects did. When cast in the condition $\times$ personality ANOVA, the condition main effect was consistently highly significant $(p<.0001)$. Finally, an interaction showed that socially anxious subjects in the experimental group were particularly attentive to the mirror $(p<.05)$.

Memory differences between the two groups were minimal, with subjects in both groups recalling about 13 of the 20 stimulus words. Also, subjects from both groups correctly answered nearly 8 of 9 questions about the thief script. Hence, group differences in performance on the judgment task cannot be attributed to differential learning about the nature of the task. Subjects in both conditions rated the task as mildly enjoyable (slightly below the midpoint on a 1-5 scale), estimated that they scored at a moderate level (mean of 4.9 hits out of 10), and rated their effort at slightly less than what they would apply to assigned reading in an elective course. On the moodadjective checklist, subjects in both conditions felt mildly mentally alert, and they did not feel particularly tense, depressed, or hostile. Main effects of testing condition did not approach significance on any of these measures.

\section{DISCUSSION}

This study demonstrates that the situational manipulation of self-focus and the arousal of test anxiety can interfere with the ability to recognize psychological implications. The main effect of testing condition is generally consistent with the prediction that self-focused attention and evaluation apprehension can interfere with the recognition of implications. However, this conclusion could be asserted more confidently had the predicted interactions with personality variables emerged.

In the absence of condition $\times$ personality interactions on the task, a number of possible explanations must be ruled out. These include (1) the possibility that subjects in the experimental group were emotionally upset by the unexpected request to be videotaped and the unveiling of the mirror; (2) the possibility that subjects in this group were distracted by looking at each other in the mirror; and (3) the possibility that these subjects read the instructions less carefully, thereby placing themselves at a disadvantage. Supplementary questionnaire data argue against the first possibility. Testing condition did not have significant effects on the mood-adjective checklist variables; if anything, the means were in the opposite direction, with the experimental group's means slightly lower on tension, depression, and hostility. As for the second possibility, it is unlikely that subjects in the experimental group were distracted by looking at each other; significant group differences did not obtain on the cognition checklist item, "[thinking about] the other people in the room." Regarding the third possibility, group differences were minimal on the thief-script memory test and on the free recall of stimulus words. Hence, these alternative explanations are not supported by the supplementary data. However, the supplementary data are consistent with the evaluation apprehension/self-focus hypothesis. Compared with subjects in the control group, those in the experimental group were more likely to think about what the experimenter thought of them and to feel that they should work more carefully. Most experimental group subjects thought about who or what was behind the mirror. The simultaneous experimental manipulation of task importance, audience, and mirror presence does not allow us to separate evaluation apprehension from self-focus. The differences in cognitions just noted imply that both processes may have been involved in the present results. Most importantly, however, the results show that the treatment did focus attention on the self and was detrimental to task performance. Obviously, a desirable next step is to separate the evaluation anxiety and self-consciousness components to determine their relative contributions to the results.

The results of the present study support our earlier conclusion that sagacity is the requisite tool for success at the word-association-judgment task. In particular, the condition main effect seems to have been mediated by a parallel effect on vigilant attention to apparent word clues. Thus, the results extend the findings of an earlier investigation (Dollinger et al., 1985) in which strategy use related to successful judgment. Moreover, the results fit reasonably well with the conceptual framework discussed by Scheibe (1979) in Mirrors, Masks, Lies, and Secrets.

Finally, the present results support another aspect of Scheibe's thesisthat the mirror metaphor, like masks, lies, and secrets, represents a means of avoiding being known and being predicted. Although our use of the mirror was more literal than metaphorical, the results are compatible 
with Scheibe's point. When the psychological judge's attention is focused on him- or herself, the person to be judged gains a psychological advantage in the interaction.

\section{REFERENCES}

Dollinger, S. J. (1985). Sagacious judgment via word association. Journal of Personality \& Social Psychology, 49, 1738-1752.

Dollinger, S. J., GreenIng, L., \& TYlenda, B. (1985). Psychologicalmindedness as "reading between the lines": Vigilance, locus of control, and sagacious judgment. Journal of Personality, 53, 603-625.

Dollinger, S. J., Reader, M. J., Marnett, J. P., \& Tylenda, B. (1983). Psychological-mindedness, psychological-construing, and the judgment of deception. Journal of General Psychology, 108, 183-191. DOLLINGER, S. J., \& Riger, A. L. (1984). On penetrating the "mask": The role of sagacity and acumen in a word-association/clinicaljudgment task. Journal of Personality \& Social Psychology, 46, 145-152.

Fenigstein, A., Scheier, M. F., \& Buss, A. H. (1975). Public and private self-consciousness: Assessment and theory. Journal of Consulting \& Clinical Psychology, 43, 522-527.

GEEN, R. G. (1976). Test anxiety, observation, and range of cue utilization. British Journal of Social \& Clinical Psychology, 15, 253-259.
GeEn, R. G. (1980). Test anxiety and cue utilization. In I. G. Sarason (Ed.), Test anxiety: Theory, research, and applications (pp. 43-61). Hillsdale, NJ: Erlbaum.

SARASON, I. G. (1972). Experimental approaches to test anxiety: Attention and the uses of information. In C. D. Spielberger (Ed.), Anxiety: Current trends in research and theory (Vol. 2, pp. 381-403). New York: Academic Press.

Sarason, I. G. (1980). Introduction to the study of test anxiety. In I. G. Sarason (Ed.), Test anxiety: Theory, research, and applications (pp. 3-14). Hillsdale, NJ: Erlbaum.

SCHEIBE, K. E. (1978). The psychologist's advantage and its nullification: Limits of human predictability. American Psychologist, 33, 869-881.

SCHEIBE, K. E. (1979). Mirrors, masks, lies, and secrets. New York: Praeger.

WINE, J. D. (1982). Evaluation anxiety: A cognitive-attentional construct. In H. W. Krohne \& L. Laux (Eds.), Achievement, stress, and anxiety (pp. 207-219). Washington, DC: Hemisphere.

ZuCKerman, M., \& LUBIN, B. (1965). Manual for the Multiple Affect Adjective Check List. San Diego: EdITS.

(Manuscript received for publication December 8, 1986.) 